\title{
Taking Mathematics to Heart
}

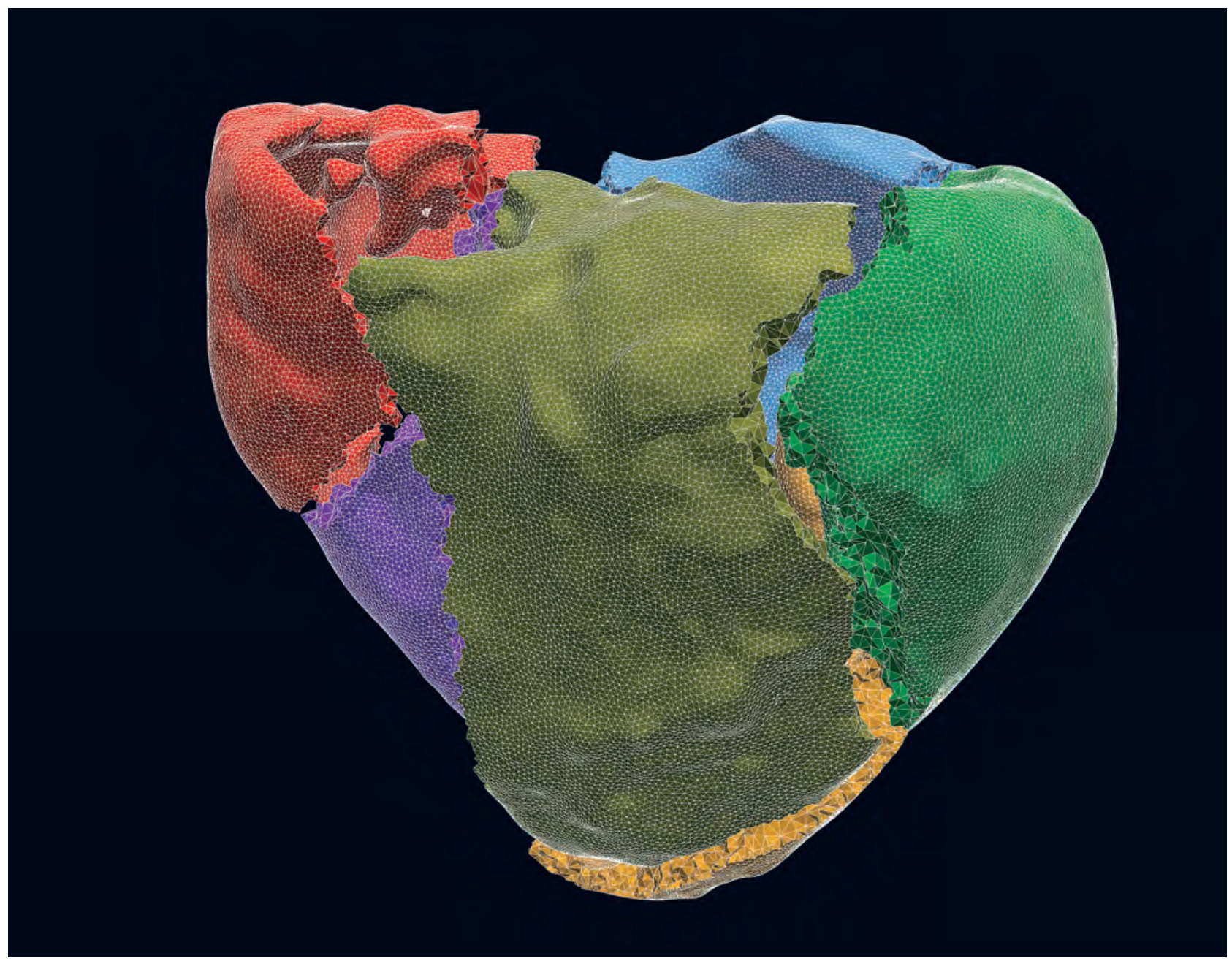

Alfio Quarteroni

\section{Introduction}

One day, a virtual version of your own heart may help doctors diagnose heart disease and determine the best treatment for you, without the need for unnecessary invasive clinical practices. The five-year ERC Advanced Grant iHEART (an integrated heart model for the simulation of the cardiac function) of the European Research Council

Alfio Quarteroni is director of the Chair of Modeling and Scientific Computing at the École Polytechnique Fédérale de Lausanne, Switzerland, and professor of numerical analysis at the Politecnico of Milan, Italy. His email address is alfio.quarteroni Qepf1.ch.

For permission to reprint this article, please contact: reprint-permission@ams.org.

DOI: http://dx.doi.org/10.1090/noti1608 has precisely the ambition to make a significant step forward in the direction of constructing a mathematical virtual heart. Its ultimate goal is to simulate heart function with increasing accuracy and to be personalized to your heart based on medical imaging (CT scans, MRI, etc.). This would hopefully help to prevent or treat cardiovascular disease by providing a personalized virtual heart to patients, essentially a detailed mathematical description of a patient's heart and how it functions-or malfunctions.

\section{The Human Heart}

The human heart is an extraordinarily complex organ that pumps an estimated 180 million liters of blood in one's lifetime, which would fill more than seven Olympic-sized 


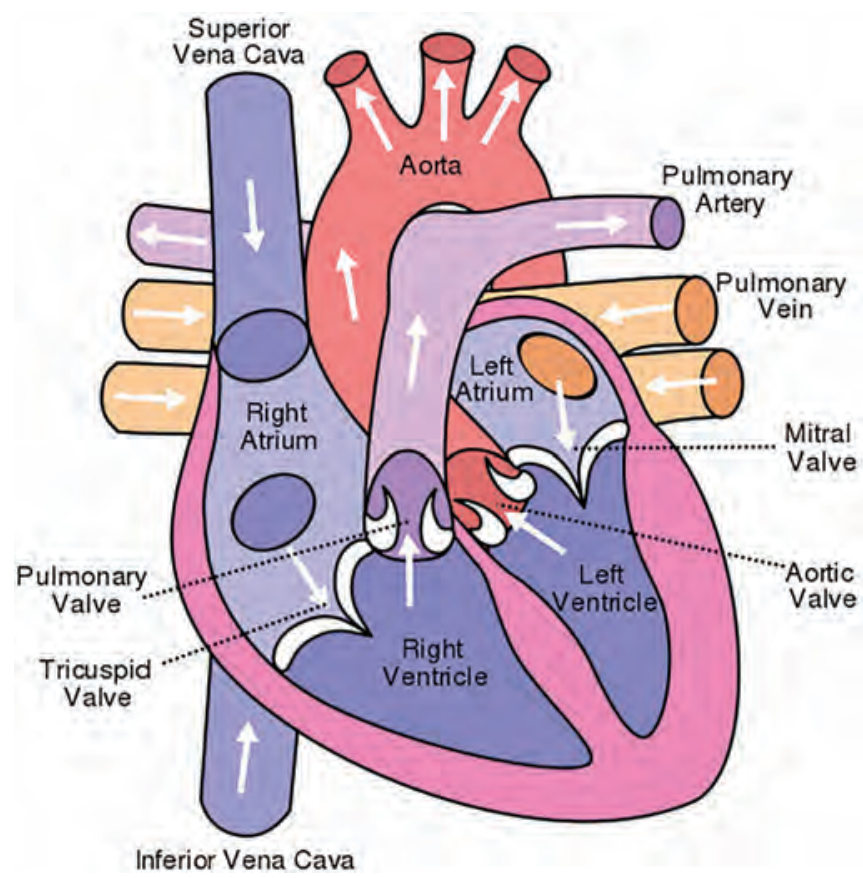

Figure 1. The heart has many working parts, but it is their synchronization that ensures the heartbeat efficiency.

swimming pools, thereby ensuring that oxygenated blood reaches the entire body.

Such an endeavor requires incredible strength and resistance: the work done by the heart to ensure every single heartbeat is comparable to that needed to tightly squeeze a tennis ball. As unreal as it appears, the heart is also the casket that guards our emotions (as already conjectured by the Greeks). The so-called "broken heart" carries some truth: after experiencing an emotionally traumatic event, our body releases stress hormones into the blood stream that can temporarily "shock" the heart and even mimic heart attack symptoms.

Cardiovascular problems may lead to malfunction, disease, or death. Heart disease causes 40 percent of deaths in the EU and costs an estimated 200 billion

Euros (over 200 billion US dolBuilding a lars) a year, yet 80 percent realistic $\quad \begin{aligned} & \text { of acquired heart diseases and } \\ & \text { stroke episodes are preventable. }\end{aligned}$ realistic Mathematics can play a great role virtual heart in improving the current availis becoming able knowledge of heart function is becoming and malfunction.

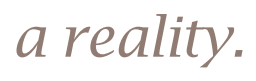

The human heart (Figure 1) is made of two atria and two ventricles, a cardiac muscle (the myocardium, surrounded by two layers, the endocardium, and the pericardium), and four valves, which undergo electrical, fluid, and mechanical processes (functions). Modeling the heart cannot be limited to the mere (albeit tremendously difficult) construction of individual core models that describe the single cardiac function, as it

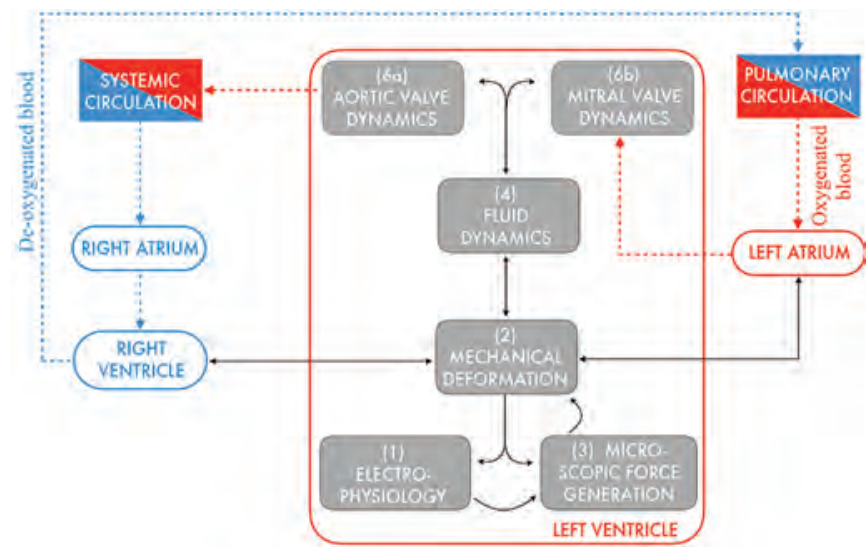

Figure 2. Sketch of the mathematical model with detail provided for the left ventricle. A mathematical model for the left ventricle requires several systems of differential equations. Each grey-shaded block represents a core cardiac model; the corresponding equations and coupling conditions are described in the text. Similar models describe the other cardiac chambers: their connection with the left ventricle is indicated by arrows; red and blue indicate oxygenated and de-oxygenated blood. Their ensemble identifies the integrated heart model (IHM).

is the beautiful harmony of their synchronization that ensures the heartbeat efficiency.

On the other side, every person's heart is unique. Correctly modeling the intricacies of each individual heart therefore requires a customizable mathematical description of both its geometry and its dynamics. But doing so in a mathematically sound way is no easy task; it requires patient-specific data and computational power to solve complex equations. Thanks to increasingly powerful computers, building a realistic virtual heart is becoming a reality.

\section{An Integrated Heart Model (IHM)}

The first cardiac models date back to the early 1970s with the seminal work of C. S. Peskin (Courant Institute) to simulate ventricular blood flow using the celebrated immersed boundary method [3]. Software development has also progressed quite rapidly in the last few years. As for today, the UT heart simulator from the group of T. Hisada (Tokyo University) [5] has provided perhaps the most comprehensive simulations of cardiac function.

Despite the scientific advances and impressive results achieved in the past forty years, a mathematically driven, fully integrated model for the entire heart function is still missing. The goal of iHEART is indeed the construction, mathematical investigation, numerical analysis, solution, data analysis, implementation into a comprehensive software library for large-scale scientific computing, model personalization, and validation on clinically relevant subject-specific cases, of an Integrated Heart Model. 


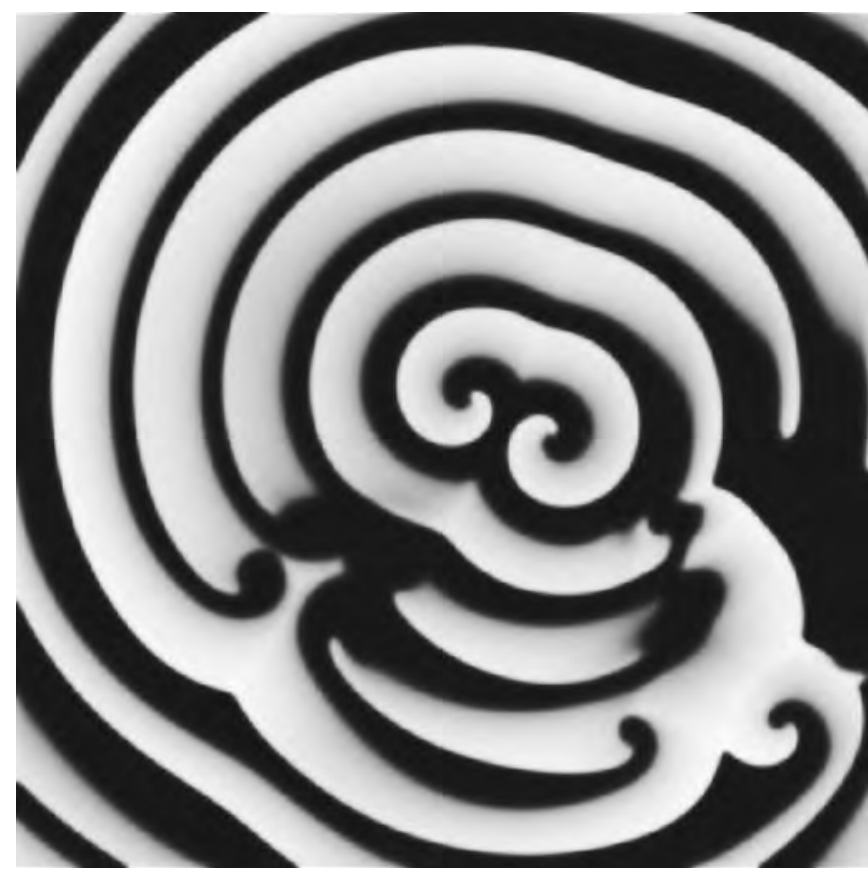

Figure 3. Wave pattern generated by the solution of the electrophysiology problem on a two-dimensional muscle specimen affected by arrhythmia.

\section{Modeling of the Core Cardiac Components}

The individual core cardiac models are represented by the electrophysiology, the passive and active mechanics of the cardiac muscle, the microscopic force generation in sarcomeres (the basic contractile units of the cardiomyocytes), the blood flow in the heart chambers, and the valve dynamics. Figure 2 shows a sketch of the models for the left ventricle and the connections with the other three heart cavities and the external circulation. Electrophysiology is the process that drives the rhythm of the heart. In the cardiac tissue, the problem at the macroscopic level is typically described by the so-called bidomain model:

$$
\left\{\begin{array}{l}
c_{m} \partial_{t} v+I_{i o n}(v, \mathbf{w}, \mathbf{c})-\nabla \cdot\left(D_{i} \nabla\left(v+u_{e}\right)\right)=0 \\
-\nabla \cdot\left(D_{i} \nabla v\right)-\nabla \cdot\left(\left(D_{i}+D_{e}\right) \nabla u_{e}\right)=I_{a p p}^{e} \\
\dot{\mathbf{w}}=\mathbf{R}_{\mathbf{w}}(v, \mathbf{w}, \mathbf{c}) \quad \dot{\mathbf{c}}=\mathbf{R}_{\mathbf{c}}(v, \mathbf{w}, \mathbf{c})
\end{array}\right.
$$

This is a system of nonlinear parabolic-elliptic PDEs coupled with a system of ODEs representing the evolution of the transmembrane potential $v=u_{i}-u_{e}$ between the intra- $u_{i}$ and extra- $u_{e}$ cellular potentials, the intracellular concentrations $c \in \mathbb{R}^{P}$ of different ionic species (in particular calcium), and the gating variables $w$ regulating transmembrane currents [1]. See Figure 3 for an example of complex wave patterns for the transmembrane potential.

Passive and active solid mechanics models provide the displacement $\mathrm{d}_{S}$ of the cardiac muscle under the action of external forces (exerted by blood flow and external tissues) and internal forces (triggered by the electrical activity:

$$
\left\{\begin{array}{l}
\rho_{S} \partial_{t t} \mathbf{d}_{S}-\nabla \cdot \sigma_{S}\left(\mathbf{d}_{S}\right)=\mathbf{0} \\
\sigma_{S}\left(\mathbf{d}_{S}\right)=\sigma_{S}\left(\mathbf{F}\left(\mathbf{d}_{S}\right), \mathbf{F}_{A}\left(\gamma_{f}\right)\right)
\end{array}\right.
$$

where $\rho_{\mathrm{s}}$ denotes the density and $\sigma_{\mathrm{s}}$ denotes the stress tensor, which depends on deformation gradient tensors $\mathrm{F}$ in turn depending on $\mathrm{d}_{S}$ and $\gamma_{f}$, the macroscopic active deformation along the fibers direction caused by the electrical activity. The passive response of the heart muscle is that of a nearly-incompressible hyperelastic transversally isotropic material comprised of mutually orthogonal fibers and collagen sheets. The orthotropic Holzapfel-Ogden constitutive law accounts for stiffening effects along the directions of fibers and sheets [4]. To model internal microscopic forces, the so-called active stress and active strain approaches are used [4]. The former considers part of the stress tensor as the active component, while the latter expresses the total solid deformation as $F=F_{E} F_{A}$, where $F_{E}$ is the deformation gradient of the passive elastic response and $F_{A}$ depends on the active deformation $\gamma_{F}$.

icroscopic force generation is the complex mechanism behind the excitation-contraction coupling between electrophysiology and active deformation, and characterizes the evolution of $\gamma_{f}$ in terms of intracellular calcium concentration (a component of c) and the muscle deformation $\mathbf{d}_{S}$, which reads:

$$
\dot{\gamma}_{f}=g_{A}\left(\gamma_{f}, \mathbf{c}, \mathbf{d}_{S}\right)
$$

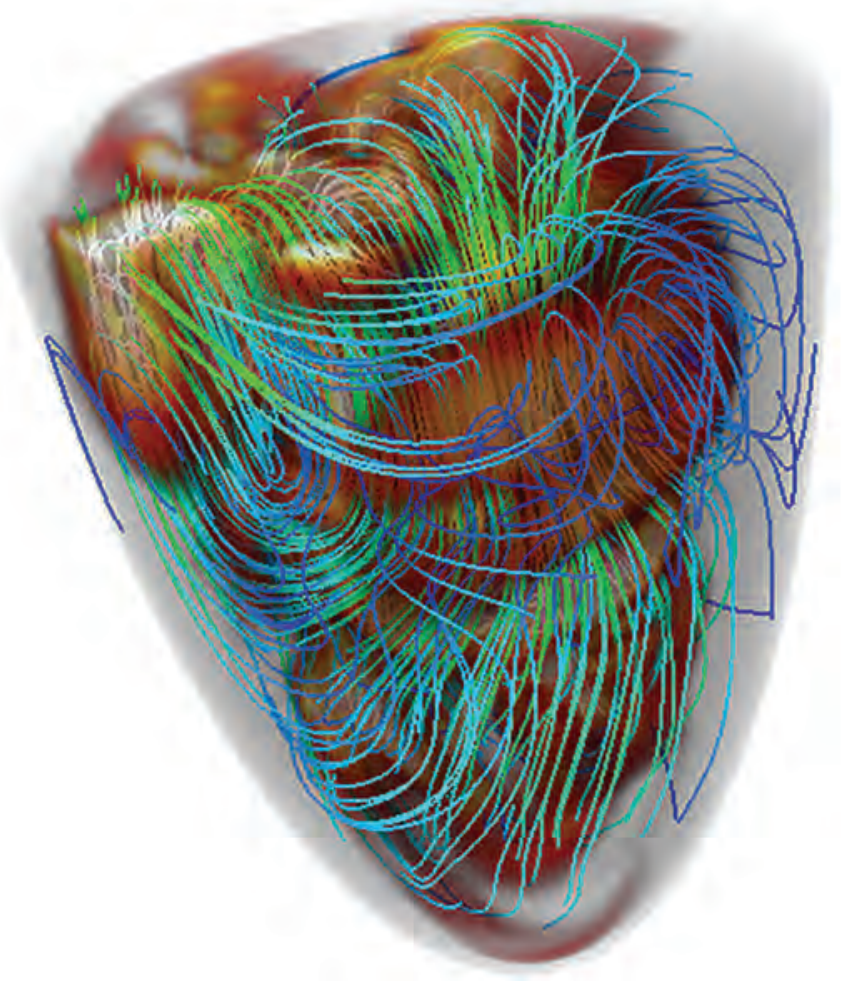

Figure 4. Velocity streamlines and vortex structures in a left ventricle. 


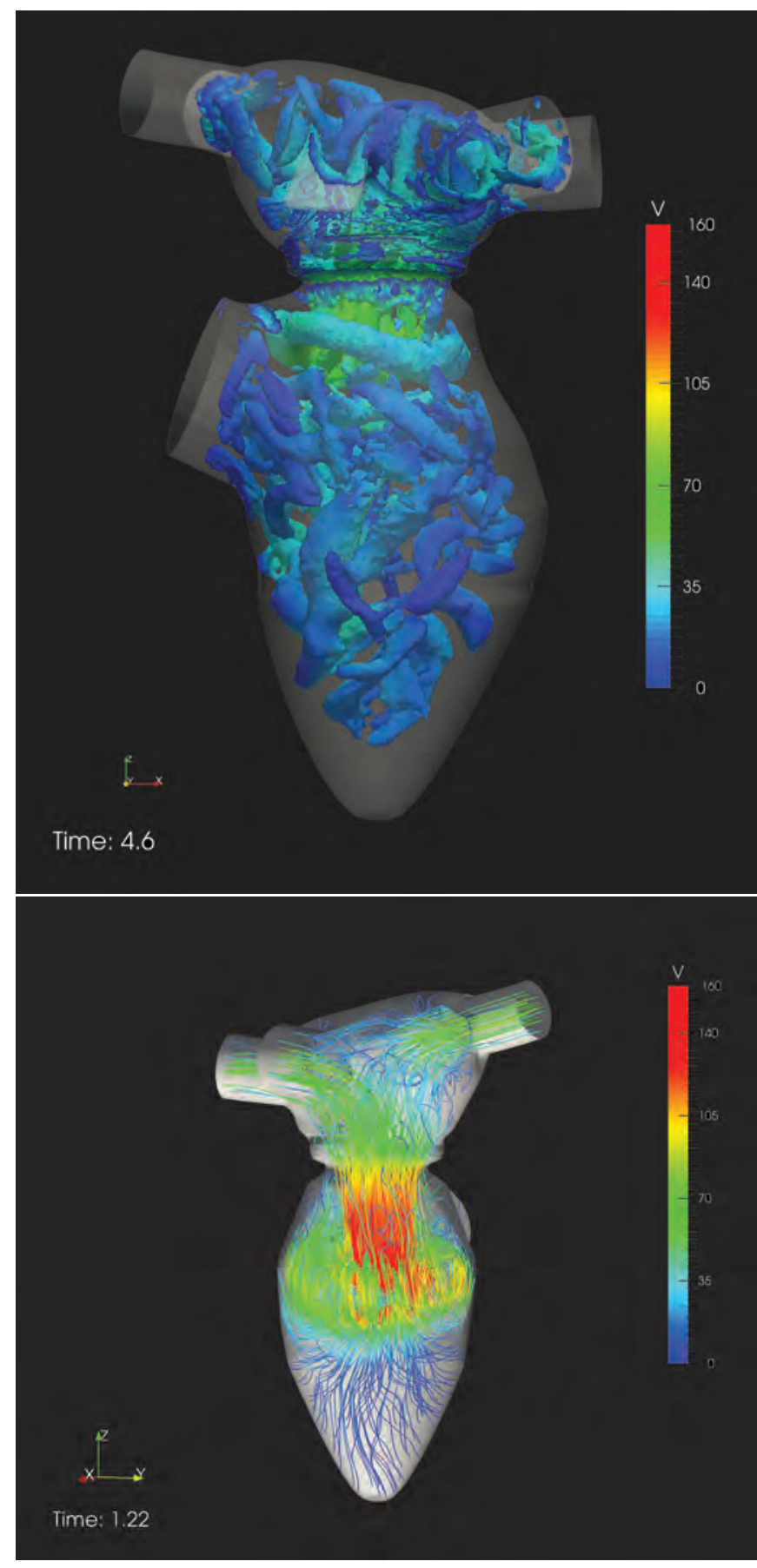

Figure 5. Computational fluid dynamics in the left heart: vortex structure and velocity streamlines in the left atrium and ventricle.

Blood flow dynamics in the heart is typically modeled by the incompressible Navier-Stokes equations. In normal conditions, the blood flow regime varies from laminar to transitional up to nearly turbulent during the heartbeat. Since each chamber undergoes large deformations, the Navier-Stokes equations can be set in an arbitrary Lagrangian-Eulerian framework. Boundary con- ditions must accommodate the interaction between blood flow and the surrounding tissue (the endocardium, including the valves), yielding a fluid-structure interaction (FSI) problem involving kinematic and dynamic fluid-solid interface conditions. See some numerical examples in Figures 4 and 5.

Valve dynamics is one of the most challenging components of heart modeling, due to their fast and complex motion. Valve response has often been represented by simple algebraic equations, ODEs in terms of leaflet displacement, 2D shells (involving the valve Cauchy stress), or as boundary conditions of the Navier-Stokes equations modeling blood flow. Moving toward a 3D valve description in the fluid domain, the immersed boundary

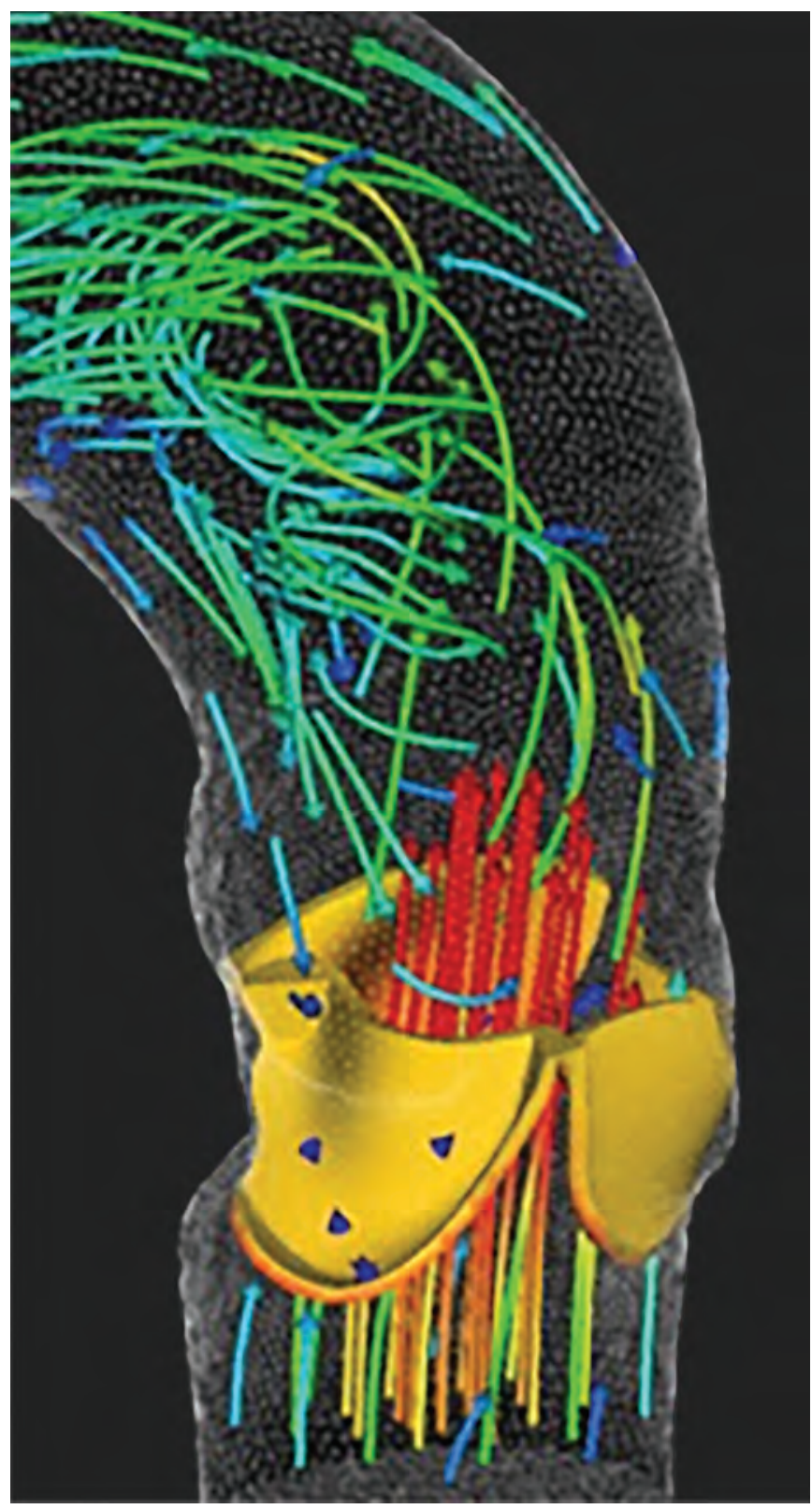

Figure 6 . Velocity streamlines in the aortic root past the tricuspid aortic valve. 


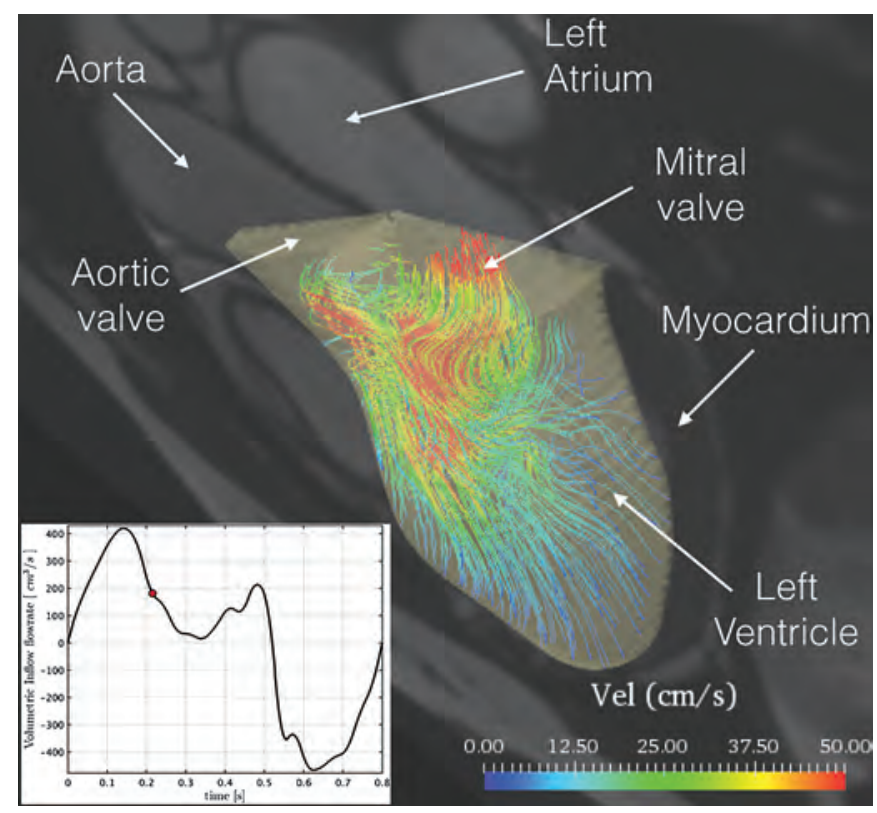

Figure 7. Flow simulation in a patient-specific left ventricle. Aortic and mitral valves are modeled through appropriate nonlinear adaptive Robin conditions.

method [3] and resistive methods [2], [4] introduce extra terms in the Navier-Stokes equations depending on Dirac masses concentrated on the valve leaflets. The position of the latter is determined by the solution of an additional mechanical problem, yielding an FSI problem. See Figures 6 and 7 for numerical simulations of blood flow in the presence of cardiac valves.

\section{Numerical Approximation of the IHM}

Regarding the numerical approximation of the IHM, properties like stability, accuracy, and efficiency of numerical methods approximating the core models are not automatically inherited by the IHM. This is even more true when using nonconforming (independent) approximations for different core components, as well as geometric multiscale strategies to accommodate for the coexistence of submodels featuring different spatial dimensions.

Coupled multiphysics problems can be approximated by either monolithic or staggered methods. With the former, all the equations are solved simultaneously; with the latter, they are solved separately with distinct solvers, often applied sequentially, a treatment that sometimes may indeed manifest time splitting errors and severe stability restrictions to the time step. Examples of numerical results obtained for the coupled electromechanics are shown in Figure 8.

Monolithic methods often employ Newton linearization, block preconditioners, and so-called Krylov iterations. The construction of efficient preconditioners is tremendously challenging and should ideally feature the properties of optimality, scalability with respect to the number of processors (for the parallel solution of largescale problems as shown in Figure 9), and robustness
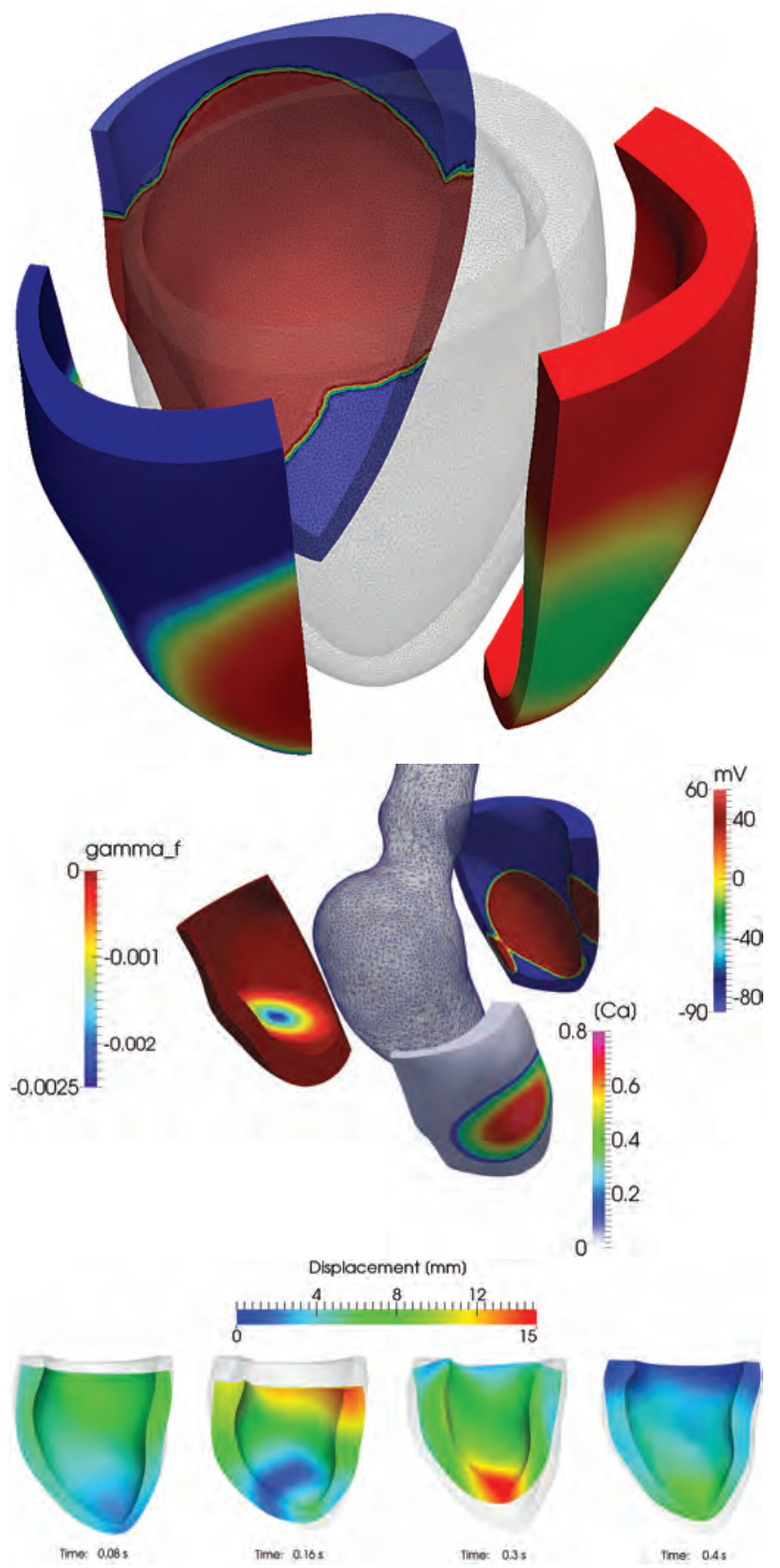

Figure 8. Numerical simulations for cardiac electromechanics in a patient-specific left ventricle; colors refer to the magnitude of the tissue displacement.

(stability with respect to the problem's parameters) in order to afford realistic and physically meaningful simulations.

\section{Addressing Variability and Uncertainty}

Patient-specific data and parameters are obtained by measurement and medical images such as magnetic resonance 


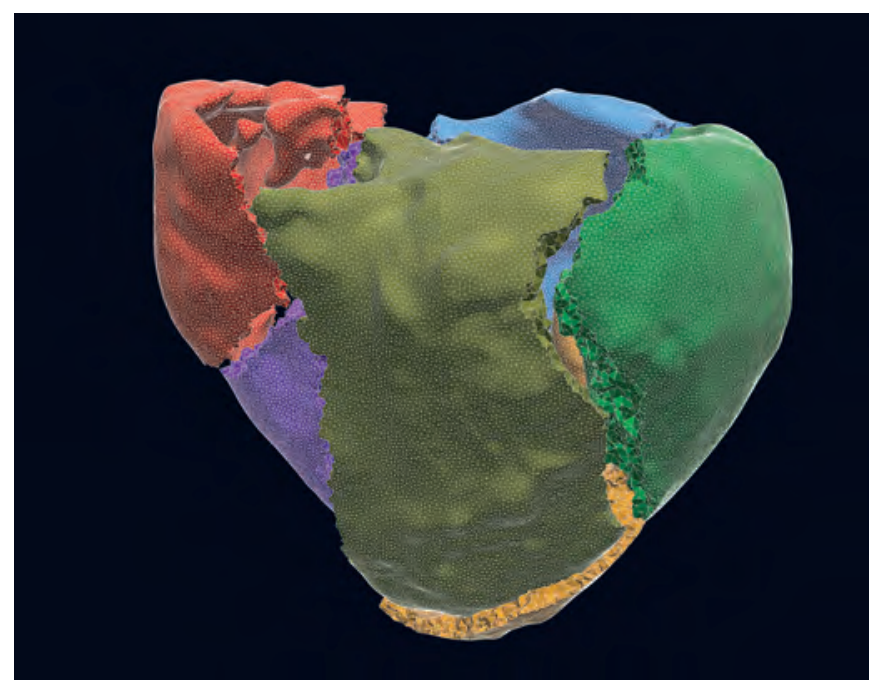

Figure 9. Multidomain partition of a 3D heart geometrical model for parallel computing. Different colors indicate different subdomains; the finite element mesh is overlaid.

imaging (MRI), computed tomography (CT), ultrasound, angiography, or Doppler echocardiography. They provide information on tissue and blood properties, pressure and flow rates determining boundary conditions, and, primarily, the shape of the heart and its chambers after image enhancement and segmentation. Data and parameters are inputs of the core cardiac models and yield parametrized systems of PDEs, whose numerical approximation for many instances of input parameters can be dramatically accelerated using reduced basis methods. Such methods exploit a suitably constructed, small dimensional linear subspace of the whole solution manifold.

Data may be insufficient, incomplete, and inaccurate. This source of uncertainty is often treated probabilistically and represented in terms of random fields to describe inter-patient variability, then propagated by a forward problem relying, e.g., on sampling techniques and multiple queries to the PDE model. Another challenging problem is that of parameter estimation, where some input quantities, not directly observable, can be identified thanks to additional clinical measurements. A possible road to parameter identification is to solve inverse problems, e.g., in a Bayesian framework, by sequential Monte Carlo methods or (extended/ensemble) Kalman filters [4].

A strict collaboration with cardiologists, cardiac surgeons, and radiologists is of paramount importance for providing a personalized virtual heart to patients, improving the understanding of heart function and dysfunction, benefiting diagnostics, decision-making for treatment, surgical planning, and design of prosthetic devices.

\section{Acknowledgments}

The contributions of EPFL MEDIACOM, L. Dedè and C. Vergara from Politecnico di Milano, and A. Manzoni from EPFL are gratefully acknowledged.

\section{References}

[1] P. Colli Franzone, L. F. PAVArino, and S. Scacchi, Mathematical Cardiac Electrophysiology, Springer, MS\&A Series, Vol. 13, 2014. MR 3308707

[2] M. Fedele, E. Faggiano, L. Dedè, and A. Quarteroni, A patient-specific aortic valve model based on moving resistive immersed implicit surfaces, Biomech. Model. Mechanobioly 2017. doi:10.1007/s10237-017-0919-1.

[3] C. S. PESKIN, The immersed boundary method, Acta Numerica 2002, 11:479-517. MR 2009378

[4] A. Quarteroni, T. Lassila, S. Rossi, and R. Ruiz-Baier, Integrated heart-coupling multiscale and multiphysics models for the simulation of the cardiac function, Comput. Methods Appl. Mech. Engrg. 2017, 314. MR 3589878

[5] S. Sugiura, T. WAshio, A. HATANo, J. OKADA, H. WATANABe, and T. HISADA, Multi-scale simulations of cardiac electrophysiology and mechanics using the University of Tokyo heart simulator, Progr. Biophys. Molecular Biol. 2012, 110.

\section{Image Credits}

Figure 1 (c) Eric Pierce. Downloaded form Wikimedia Commons and used under the Creative Commons Attribution-Share Alike 3.0 Unported License.

Figures 2, 3, and 9 courtesy of CMCS, EPFL.

Figure 4 numerical simulation by A. Tagliabue, EPFL.

Figure 5 numerical simulation by F. Menghini, EPFL.

Figure 6 numerical simulation by M. Fedele and E. Faggiano, Politecnico di Milano, visualization by Jean Favre from CSCS, Lugano.

Figure 7 numerical simulation by A. Tagliabue, EPFL.

Figure 8 numerical simulation by A. Gerbi, EPFL.

Author photo courtesy of Alfio Quarteroni.

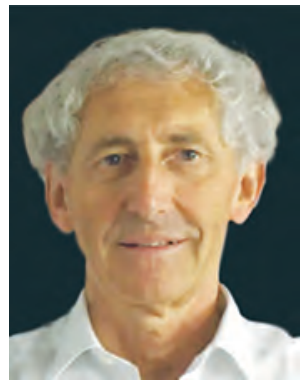

Alfio Quarteroni

\section{ABOUT THE AUTHOR}

Alfio Quarteroni, Euler Vorlesung lecturer in 2017 and winner of the International Galileo Galilei prize for Sciences in 2015, is author of twenty-two books and more than 350 papers in numerical modeling of fluid mechanics, geophysics, medicine, and sports. His group has carried out the mathematical simulation for the Alinghi yacht, winner of two editions of the America's Cup. 